\title{
Higher loop renormalization of fermion bilinear operators
}

\author{
A. Skouroupathis* ${ }^{\dagger \dagger}$ and H. Panagopoulos \\ University of Cyprus, Department of Physics, Cyprus \\ E-mail: php4as01@ucy.ac.cy, haris@ucy.ac.cy
}

We compute the two-loop renormalization functions, in the RI' scheme, of local bilinear quark operators $\bar{\psi} \Gamma \psi$, where $\Gamma$ denotes the Scalar and Pseudoscalar Dirac matrices, in the lattice formulation of QCD. We consider both the flavor non-singlet and singlet operators; the latter, in the scalar case, leads directly to the two-loop fermion mass renormalization, $Z_{m}$.

As a prerequisite for the above, we also compute the quark field renormalization, $Z_{\psi}$, up to two loops.

We use the clover action for fermions and the Wilson action for gluons. Our results are given as a polynomial in $c_{S W}$, in terms of both the renormalized and bare coupling constant, in the renormalized Feynman gauge. We also confirm the 1-loop renormalization functions, for generic gauge.

A longer write-up of the present work, including the conversion of our results to the $\overline{M S}$ scheme and a generalization to arbitrary fermion representations, can be found in arXiv:0707.2906.

The XXV International Symposium on Lattice Field Theory

July 30-4 August 2007

Regensburg, Germany

\footnotetext{
*Speaker.

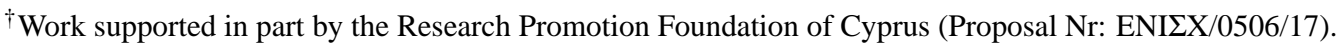




\section{Introduction}

Studies of hadronic properties using the lattice formulation of QCD rely on the computation of matrix elements and correlation functions of composite operators, made out of quark fi elds. A whole variety of such operators has been considered and studied in numerical simulations, including local and extended bilinears, and four-fermi operators. A proper renormalization of these operators is most often indispensable for the extraction of physical results from the lattice.

In this work we study the renormalization of fermion bilinears $\mathscr{O}=\bar{\psi} \Gamma \psi$ on the lattice, where $\Gamma=\hat{1}, \gamma_{5}$. We consider both flavor singlet and nonsinglet operators. The cases $\Gamma=\gamma_{\mu}, \gamma_{5} \gamma_{\mu}, \gamma_{5} \sigma_{\mu \nu}$, will be presented in a sequel to this work. In order to obtain the renormalization functions of fermion bilinears we also compute the quark fi eld renormalization, $Z_{\psi}$, as a prerequisite.

We employ the standard Wilson action for gluons and clover-improved Wilson fermions. The number of quark flavors $N_{f}$, the number of colors $N_{c}$ and the clover coeffi cient $c_{S W}$ are kept as free parameters. Our two-loop calculations have been performed in the bare and in the renormalized Feynman gauge. For 1-loop quantities, the gauge parameter is allowed to take arbitrary values.

The main results presented in this work are the following 2-loop bare Green's functions (amputated, one-particle irreducible (1PI)): • Fermion self-energy: $\Sigma_{\psi}^{L}\left(q, a_{\mathrm{L}}\right), \bullet 2$-pt function of the scalar $\bar{\psi} \psi: \Sigma_{S}^{L}\left(q a_{\mathrm{L}}\right), \bullet 2$-pt function of the pseudoscalar $\bar{\psi} \gamma_{5} \psi: \Sigma_{P}^{L}\left(q a_{\mathrm{L}}\right)\left(a_{\mathrm{L}}:\right.$ lattice spacing, $q$ : external momentum)

In general, one can use bare Green's functions to construct $Z_{\mathscr{O}}^{X, Y}$, the renormalization function for operator $\mathscr{O}$, computed within a regularization $X$ and renormalized in a scheme $Y$. We employ the $R I^{\prime}$ scheme to compute the various renormalization functions: $Z_{\psi}^{L, R I^{\prime}}, Z_{S}^{L, R I^{\prime}}, Z_{P}^{L, R I^{\prime}}$. The corresponding quantities in the $\overline{M S}$ scheme: $Z_{\psi}^{L, \overline{M S}}, Z_{S}^{L, \overline{M S}}, Z_{P}^{L, \overline{M S}}$, are presented in the longer write-up [1].

The flavor singlet scalar renormalization function is equal to the fermion mass multiplicative renormalization, $Z_{m}$, which is an essential ingredient in computing quark masses.

Finally, as one of several checks on our results, we construct the 2-loop renormalized Green's functions in $R I^{\prime}: \Sigma_{\mathscr{O}}^{R I^{\prime}}(q, \bar{\mu})(\mathscr{O} \equiv \psi, S, P)$, as well as their counterparts in $\overline{M S}: \Sigma_{\mathscr{O}}^{\overline{M S}}(q, \bar{\mu})$. The values of all these functions, computed on the lattice, coincide with values computed in dimensional regularization (as can be inferred, e.g., from [2]).

The present work is the fi rst two-loop computation of the renormalization of fermion bilinears on the lattice. There have been made several attempts to estimate $Z_{\mathscr{O}}$ non-perturbatively; see Ref.[1] for a list of recent references. Some results have also been obtained using stochastic perturbation theory [3]. A related computation, regarding the fermion mass renormalization $Z_{m}$ with staggered fermions can be found in [何.

\section{Formulation of the problem}

Lattice action: We will make use of the Wilson formulation of the QCD action on the lattice, with the addition of the clover (SW) term for fermions. The clover coeffi cient $c_{S W}$ in the Langrangian is treated here as a free parameter; The fermion mass $m_{\mathrm{o}}$ in the Langrangian is a free parameter. However, since we will be using mass independent renormalization schemes, all renormalization 
functions which we will be calculating, must be evaluated at vanishing renormalized mass, that is, when $m_{\mathrm{o}}$ is set equal to the critical value $m_{\mathrm{cr}}: m_{\mathrm{o}} \rightarrow m_{\mathrm{cr}}=0+\mathscr{O}\left(g_{\mathrm{o}}^{2}\right)$.

Definition of renormalized quantities: As a prerequisite, we will need the renormalization functions for the gluon, ghost and fermion fi elds $\left(A_{\mu}^{a}, c^{a}, \psi\right)$, and for the coupling constant $g$ and gauge parameter $\alpha$, defi ned as follows:

$$
A_{\mu \mathrm{o}}^{a}=\sqrt{Z_{A}} A_{\mu}^{a}, c_{\mathrm{o}}^{a}=\sqrt{Z_{c}} c^{a}, \psi_{\mathrm{o}}=\sqrt{Z_{\psi}} \psi, g_{\mathrm{o}}=\mu^{\varepsilon} Z_{g} g, \alpha_{\mathrm{o}}=Z_{a}^{-1} Z_{A} \alpha
$$

The scale $\mu$ enters the relation between $g_{o}$ and $g$ only in dimensional regularization $(D=4-2 \varepsilon$ dimensions). We will need $Z_{A}, Z_{c}, Z_{\alpha}$ and $Z_{g}$ to 1 loop and $Z_{\psi}$ to 2 loops.

Definition of the RI' scheme: This renormalization scheme [7], 8, 的 is more immediate for a lattice regularized theory. It is defi ned by imposing a set of normalization conditions on matrix elements at a scale $\bar{\mu}$, where (just as in the $\overline{M S}$ scheme): $\bar{\mu}=\mu\left(4 \pi / \mathrm{e}^{\gamma_{\mathrm{E}}}\right)^{1 / 2} \quad\left(\gamma_{\mathrm{E}}\right.$ is the Euler constant $)$

In Euclidean space, the fermion self energy $\Sigma_{\psi}^{L}\left(q, a_{\mathrm{L}}\right)=i q+m_{\mathrm{o}}+\mathscr{O}\left(g_{o}^{2}\right)$ is renormalized by:

$$
\lim _{a_{\mathrm{L}} \rightarrow 0}\left[Z_{\psi}^{L, R I^{\prime}}\left(a_{\mathrm{L}} \bar{\mu}\right) \operatorname{tr}\left(\Sigma_{\psi}^{L}\left(q, a_{\mathrm{L}}\right) q\right) /\left(4 i q^{2}\right)\right]_{q^{2}=\bar{\mu}^{2}}=1
$$

The trace here is over Dirac indices; a Kronecker delta in color and in flavor indices has been factored out of the defi nition of $\Sigma_{\psi}^{L}$. Similar conditions hold for $Z_{c}$ (extracted from the ghost self energy) and $Z_{A}, Z_{\alpha}$ (extracted from the gluon propagator). We have checked explicitly that $Z_{\alpha}^{L, R I^{\prime}}=1$ up to one loop, in agreement with the continuum.

For consistency with the Slavnov-Taylor identities, $Z_{g}$ in the $R I^{\prime}$ scheme is defi ned as in the $\overline{M S}$ scheme. In dimensional regularization $(D R)$ this is achieved by tuning the value of $Z_{g}$ in such a way as to absorb only the poles in $\varepsilon$ which appear in the gluon-fermion-antifermion 1PI vertex function $G_{A \bar{\psi} \psi}$ (together with matching powers of $\ln (4 \pi)-\gamma_{\mathrm{E}}$ ); this leads to a result for $G_{A \bar{\psi} \psi}^{\mathrm{fin} \psi}$ which is fi nite but not unity. Alternatively, a similar procedure can be performed on the gluonghost-antighost vertex $G_{A^{-}{ }^{c} \varepsilon}$ leading to exactly the same value for $Z_{g}$.

The corresponding renormalization condition can be applied on the lattice vertex, $G_{A \bar{\psi} \psi}^{L}$, requiring that the renormalized expression $G_{A \bar{\psi} \psi}^{\mathrm{fi}}$ ite is the same as that stemming from DR, and similarly for $G_{A^{-} c c}^{L}$ We have calculated $Z_{g}^{L, R I^{\prime}}$, using either one of $G_{A^{-} c c}^{L}$ or $G_{A \bar{\psi} \psi}^{L}$, and have verifi ed that the same result is obtained.

Renormalization of fermion bilinears: The renormalization of lattice operators $\mathscr{O}_{\Gamma}=\bar{\psi} \Gamma \psi$ is defi ned by: $\mathscr{O}_{\Gamma}^{R I^{\prime}}=Z_{\Gamma}^{L, R I^{\prime}}\left(a_{\mathrm{L}} \bar{\mu}\right) \mathscr{O}_{\Gamma \mathrm{o}}$

For the scalar (S) and pseudoscalar (P) operators, $Z_{\Gamma}^{L, R I^{\prime}}$ can be obtained through the corresponding bare 2-point functions $\Sigma_{\Gamma}^{L}\left(q a_{\mathrm{L}}\right)$ (amputated, 1PI) on the lattice, in the following way:

$$
\lim _{a_{\mathrm{L}} \rightarrow 0}\left[Z_{\psi}^{L, R I^{\prime}} Z_{S}^{L, R I^{\prime}} \Sigma_{S}^{L}\left(q a_{\mathrm{L}}\right)\right]_{q^{2}=\bar{\mu}^{2}}=\hat{1}, \lim _{a_{\mathrm{L}} \rightarrow 0}\left[Z_{\psi}^{L, R I^{\prime}} Z_{P}^{L, R I^{\prime}} \Sigma_{P}^{L}\left(q a_{\mathrm{L}}\right)\right]_{q^{2}=\bar{\mu}^{2}}=\gamma_{5}
$$

Once $Z_{\Gamma}^{L, R I^{\prime}}$ have been calculated, one may convert them to the $\overline{M S}$ scheme (see Ref.[1]).

\section{Computation and Results}

There are 28 Feynman diagrams contributing to the fermion self-energy $\Sigma_{\psi}^{L}\left(q, a_{\mathrm{L}}\right)$ at 1 and 2 loops, and 21 diagrams contributing to $\Sigma_{S}^{L}\left(q a_{\mathrm{L}}\right), \Sigma_{P}^{L}\left(q a_{\mathrm{L}}\right)$. For flavor singlet bilinears, there are 4 extra diagrams, shown in Fig.1, in which the operator insertion occurs inside a closed fermion loop. 
The evaluation and algebraic manipulation of Feynman diagrams, leading to a code for numerical loop integration, is performed automatically using our software for Lattice Perturbation Theory, written in Mathematica. The most laborious aspect of the procedure is the extraction of the dependence on the external momentum $q$. This is a delicate task at two loops; for this purpose, we cast algebraic expressions (typically involving thousands of summands) into terms which can be naively Taylor expanded in $q$ to the required order, plus a smaller set of terms containing superfi cial divergences and/or subdivergences. The latter can be evaluated by analytical continuation to $D>4$ dimensions, and splitting each expression into a UV-fi nite part (which can thus be calculated in the continuum), and a part which is polynomial in $q$.

Some of the diagrams contributing to $\Sigma_{\psi}^{L}\left(q, a_{\mathrm{L}}\right), \Sigma_{S}^{L}\left(q a_{\mathrm{L}}\right)$ and $\Sigma_{P}^{L}\left(q a_{\mathrm{L}}\right)$ are infrared divergent when considered separately, and thus must be grouped together in order to give fi nite results, for example, diagrams (1-2), (3-4) in Fig.1. As mentioned before, all calculations should be performed at vanishing renormalized mass; this can be achieved by working with massless fermion propagators, provided an appropriate fermion mass counterterm is introduced on 1-loop diagrams.

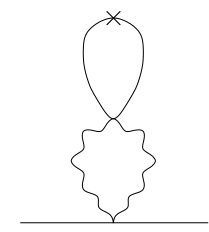

1

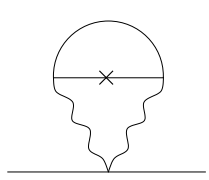

2

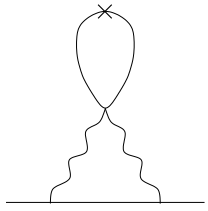

3

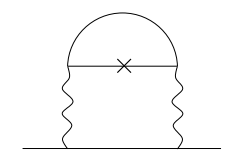

4

FIG. 1. Extra two-loop diagrams contributing to $Z_{S, \text { singlet }}$ A cross denotes an insertion of a flavor singlet operator. Wavy (solid) lines represent gluons (fermions)

All two-loop diagrams have been calculated in the bare Feynman gauge $\left(\alpha_{\mathrm{o}}=1\right)$. One-loop diagrams have been calculated for generic values of $\alpha_{0}$; this allows us to convert our two-loop results to the renormalized Feynman gauge $\left(\alpha_{R I^{\prime}}=1\right.$ or $\left.\alpha_{\overline{M S}}=1\right)$.

Numerical loop integration was carried out by our "integrator" program, a metacode written in Mathematica, for converting lengthy integrands into effi cient Fortran code. Two-loop integrals were evaluated for lattices of size up to $L=40$; the results were then extrapolated to $L \rightarrow \infty$. The extrapolation error can be estimated quite accurately (see, e.g. Ref. [10]), given that $L$-dependence of results can only span a restricted set of functional forms.

Our 1-loop results for $Z_{\psi}, Z_{S}$ and $Z_{P}$ confi rm existing results [9] (there is, however, a difference in $Z_{P}^{L, \overline{M S}}$, due to an extra fi nite renormalization factor, $Z_{5}$, which is required in $\overline{M S}$ [1]).

Two-loop results: The evaluation of all Feynman diagrams leads directly to the bare Green's functions $\Sigma_{\psi}^{L}, \Sigma_{S}^{L}$ and $\Sigma_{P}^{L}$. These, in turn, can be converted to the corresponding renormalization functions $Z_{\psi}^{L, Y}, Z_{S}^{L, Y}$ and $Z_{P}^{L, Y}\left(Y=R I^{\prime}\right.$ or $\overline{M S}$ ), via Eqs.(2.2) and (2.3). To this end, we need the one-loop expression for $Z_{A}^{L, R I^{\prime}}$. To express our results in terms of the renormalized $g$, we also need the one-loop expression for $Z_{g}^{L, R I^{\prime}}$. Our 1-loop computation of $Z_{A}^{L, R I^{\prime}}$ and $Z_{g}^{L, R I^{\prime}}$ is in agreement with older references (see e.g. [6]). We present below $Z_{\psi}^{L, R I^{\prime}}, Z_{S}^{L, R I^{\prime}}$ and $Z_{P}^{L, R I^{\prime}}$ to two loops in the renormalized Feynman gauge $\alpha_{R I^{\prime}}=1$. Bare Green's functions can be easily recovered from the corresponding $Z$ 's. The errors result from the $L \rightarrow \infty$ extrapolation. We employ a standard normalization for the algebra generators: $\operatorname{tr}\left(T^{a} T^{b}\right)=\delta^{a b} / 2 .\left(a_{\mathrm{o}} \equiv g_{\mathrm{o}}^{2} / 16 \pi^{2}, c_{F} \equiv\left(N_{c}^{2}-1\right) /\left(2 N_{c}\right)\right)$ 


$$
\begin{aligned}
& Z_{\psi}^{L, R I^{\prime}}=1+a_{\mathrm{o}} c_{F}\left[\ln \left(a_{\mathrm{L}}^{2} \bar{\mu}^{2}\right)+11.852404288(5)-2.248868528(3) c_{\mathrm{SW}}-1.397267102(5) c_{\mathrm{SW}}^{2}\right] \\
& +a_{\mathrm{o}}^{2} c_{F}\left[\ln ^{2}\left(a_{\mathrm{L}}^{2} \bar{\mu}^{2}\right)\left(\frac{1}{2} c_{F}+\frac{2}{3} N_{f}-\frac{8}{3} N_{c}\right)\right. \\
& +\ln \left(a_{\mathrm{L}}^{2} \bar{\mu}^{2}\right)\left(-6.36317446(8) N_{f}+0.79694523(2) N_{f} c_{\mathrm{SW}}-4.712691443(4) N_{f} c_{\mathrm{SW}}^{2}\right. \\
& \left.+49.83082185(5) c_{F}-2.24886861(7) c_{F} c_{\mathrm{SW}}-1.39726705(1) c_{F} c_{\mathrm{SW}}^{2}+29.03029398(4) N_{c}\right) \\
& +N_{f}\left(-7.838(2)+1.153(1) c_{\mathrm{SW}}+3.202(3) c_{\mathrm{SW}}^{2}+6.2477(6) c_{\mathrm{SW}}^{3}+4.0232(6) c_{\mathrm{SW}}^{4}\right) \\
& +c_{F}\left(505.39(1)-58.210(9) c_{\mathrm{SW}}+20.405(5) c_{\mathrm{SW}}^{2}+18.8431(8) c_{\mathrm{SW}}^{3}+4.2793(2) c_{\mathrm{SW}}^{4}\right) \\
& \left.+N_{c}\left(-20.59(1)-3.190(5) c_{\mathrm{SW}}-23.107(6) c_{\mathrm{SW}}^{2}-5.7234(5) c_{\mathrm{SW}}^{3}-0.7938(1) c_{\mathrm{SW}}^{4}\right)\right] \\
& Z_{S}^{L, R I^{\prime}}=1+a_{\mathrm{o}} c_{F}\left[3 \ln \left(a_{\mathrm{L}}^{2} \bar{\mu}^{2}\right)-17.9524103(1)-7.7379159(3) c_{\mathrm{SW}}+1.38038065(4) c_{\mathrm{SW}}^{2}\right] \\
& +a_{\mathrm{o}}^{2} c_{F}\left[\ln ^{2}\left(a_{\mathrm{L}}^{2} \bar{\mu}^{2}\right)\left(\frac{9}{2} c_{F}+N_{f}-\frac{11}{2} N_{c}\right)\right. \\
& +\ln \left(a_{\mathrm{L}}^{2} \bar{\mu}^{2}\right)\left(-8.1721694(5) N_{f}+2.3908354(3) N_{f} c_{\mathrm{SW}}-14.13807433(4) N_{f} c_{\mathrm{SW}}^{2}\right. \\
& \left.+66.0780218(9) c_{F}-23.213749(2) c_{F} c_{\mathrm{SW}}+4.1411425(3) c_{F} c_{\mathrm{SW}}^{2}+55.7975008(9) N_{c}\right) \\
& +N_{f}\left(24.003(3)+11.878(5) c_{\mathrm{SW}}+25.59(1) c_{\mathrm{SW}}^{2}+22.078(3) c_{\mathrm{SW}}^{3}-6.1807(8) c_{\mathrm{SW}}^{4}\right) \\
& +c_{F}\left(-602.35(6)+66.80(7) c_{\mathrm{SW}}+75.42(5) c_{\mathrm{SW}}^{2}-27.759(4) c_{\mathrm{SW}}^{3}-2.688(1) c_{\mathrm{SW}}^{4}\right) \\
& \left.+N_{c}\left(-38.16(4)-120.26(5) c_{\mathrm{SW}}-16.18(3) c_{\mathrm{SW}}^{2}+12.576(3) c_{\mathrm{SW}}^{3}+1.0175(8) c_{\mathrm{SW}}^{4}\right)\right] \\
& Z_{P}^{L, R I^{\prime}}=1+a_{\mathrm{o}} c_{F}\left[3 \ln \left(a_{\mathrm{L}}^{2} \bar{\mu}^{2}\right)-27.5954414(1)+2.248868528(3) c_{\mathrm{SW}}-2.03601561(4) c_{\mathrm{SW}}^{2}\right] \\
& +a_{\mathrm{o}}^{2} c_{F}\left[\ln ^{2}\left(a_{\mathrm{L}}^{2} \bar{\mu}^{2}\right)\left(\frac{9}{2} c_{F}+N_{f}-\frac{11}{2} N_{c}\right)\right. \\
& +\ln \left(a_{\mathrm{L}}^{2} \bar{\mu}^{2}\right)\left(-8.1721694(4) N_{f}+2.39083540(6) N_{f} c_{\mathrm{SW}}-14.13807433(4) N_{f} c_{\mathrm{SW}}^{2}\right. \\
& \left.+37.1489292(7) c_{F}+6.746606(1) c_{F} c_{\mathrm{SW}}-6.1080465(3) c_{F} c_{\mathrm{SW}}^{2}+55.7975008(7) N_{c}\right) \\
& +N_{f}\left(38.231(3)-7.672(5) c_{\mathrm{SW}}+55.32(1) c_{\mathrm{SW}}^{2}-7.049(3) c_{\mathrm{SW}}^{3}+4.7469(8) c_{\mathrm{SW}}^{4}\right) \\
& +c_{F}\left(-876.98(4)+85.80(2) c_{\mathrm{SW}}+37.37(4) c_{\mathrm{SW}}^{2}+19.974(3) c_{\mathrm{SW}}^{3}+2.873(1) c_{\mathrm{SW}}^{4}\right) \\
& \left.+N_{c}\left(-104.35(3)-38.70(2) c_{\mathrm{SW}}-13.93(3) c_{\mathrm{SW}}^{2}-4.429(2) c_{\mathrm{SW}}^{3}-1.2898(7) c_{\mathrm{SW}}^{4}\right)\right]
\end{aligned}
$$

All expressions reported thus far for $Z_{S}$ and $Z_{P}$ refer to flavor non singlet operators. In the case of $Z_{P}$, all diagrams of Fig.1 vanish, so that singlet and non singlet results coincide. $Z_{S}$ on the other hand, receives an additional fi nite contribution (which is the same for the $\overline{M S}$ scheme as well):

$$
\begin{aligned}
Z_{S, \text { singlet }}^{L, R I^{\prime}}=Z_{S}^{L, R I^{\prime}}+a_{\mathrm{o}}^{2} c_{F} N_{f}( & -107.76(1)+82.27(2) c_{\mathrm{SW}}-29.727(4) c_{\mathrm{SW}}^{2} \\
& \left.+3.4400(7) c_{\mathrm{SW}}^{3}+2.2758(4) c_{\mathrm{SW}}^{4}\right)
\end{aligned}
$$


In Figs. 2, 3 and 4 we plot $Z_{\psi}^{L, R I^{\prime}}, Z_{S}^{L, R I^{\prime}}$ and $Z_{P}^{L, R I^{\prime}}$, respectively, as a function of $c_{\mathrm{SW}}$. For defi niteness, we have set $N_{c}=3, \bar{\mu}=1 / a_{\mathrm{L}}$ and $\beta_{\mathrm{o}} \equiv 2 N_{c} / g_{\mathrm{o}}^{2}=6.0$. Our results up to two loops for each $Z$ are shown for both $N_{f}=0$ and $N_{f}=2$, and compared to the corresponding one-loop results. Furthermore, in the scalar case, we also present the two-loop result for the flavor singlet operator.

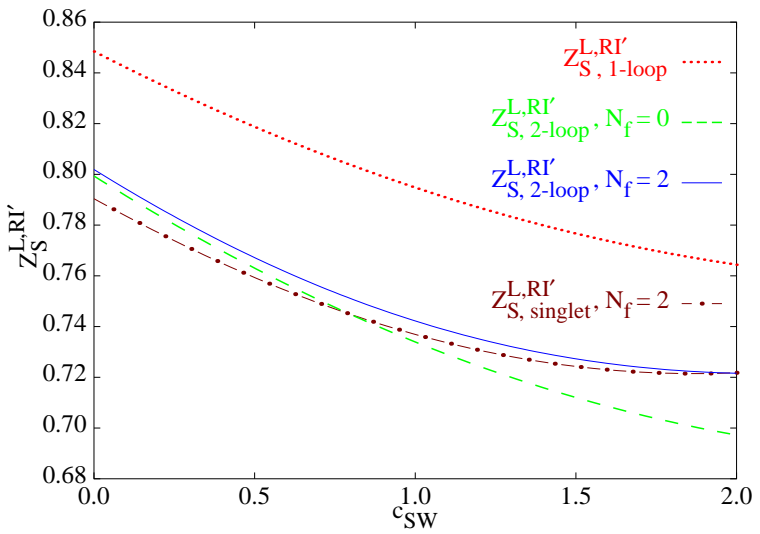

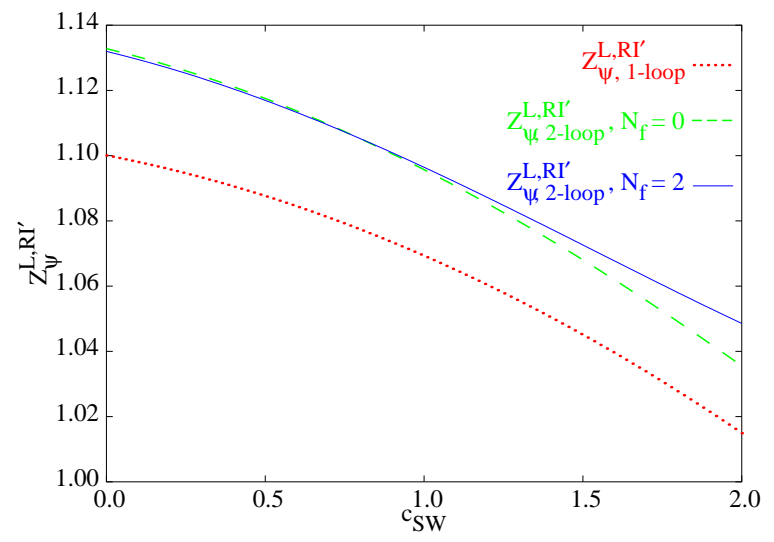

FIG. 2. $Z_{\psi}^{L, R I^{\prime}}\left(a_{\mathrm{L}} \bar{\mu}\right)$ vs. $c_{\mathrm{SW}}\left(N_{c}=3, \bar{\mu}=1 / a_{\mathrm{L}}\right.$, $\beta_{\mathrm{o}}=6.0$ ). Results up to 2 loops are shown for $N_{f}=0$ (dashed line) and $N_{f}=2$ (solid line); one-loop results are plotted with a dotted line.

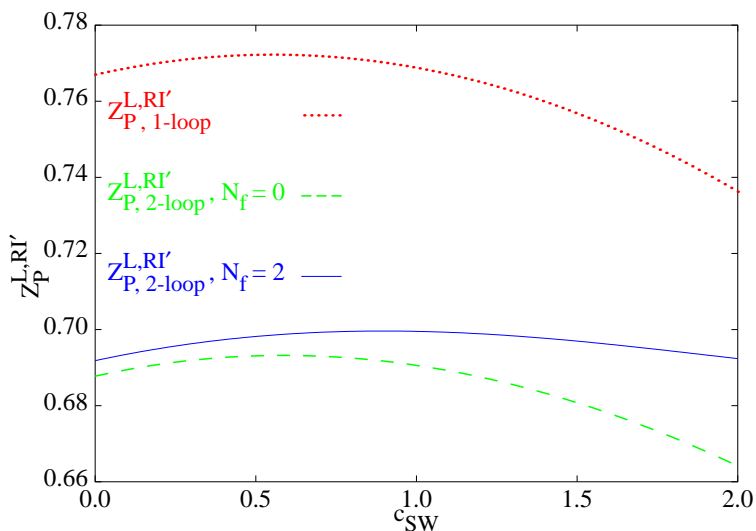

FIGS. 3, 4. $Z_{S}^{L, R I^{\prime}}\left(a_{\mathrm{L}} \bar{\mu}\right), Z_{P}^{L, R I^{\prime}}\left(a_{\mathrm{L}} \bar{\mu}\right)$ vs. $c_{\mathrm{SW}}\left(N_{c}=3, \bar{\mu}=1 / a_{\mathrm{L}}, \beta_{\mathrm{o}}=6.0\right)$. Results up to 2 loops, for the flavor non-singlet, are shown for $N_{f}=0$ (dashed line) and $N_{f}=2$ (solid line); 2-loop results for the scalar flavor singlet, for $N_{f}=2$, are plotted with a dash-dotted line; one-loop results are plotted with a dotted line.

\section{Discussion}

As can be seen from Figs.2-4, all 2-loop renormalization functions differ from 1-loop values in a signifi cant way; this difference should be taken into account in MC simulations, in order to reduce systematic error. At the same time, 2-loop contributions are consistently smaller than 1-loop contributions, indicating that the (asymptotic) perturbative series are under control.

The dependence on the clover parameter $c_{\mathrm{SW}}$ is also quite pronounced. In the present work, $c_{\mathrm{SW}}$ was left as a free parameter; its optimal value, as dictated by $\mathscr{O}\left(a_{\mathrm{L}}\right)$ improvement, has been estimated both non-perturbatively and perturbatively (to 1-loop) in the literature.

Our results regard both the flavor nonsinglet and singlet operators. For the pseudoscalar operator, these cases coincide, just as in dimensional regularization. The scalar, on the other hand, receives an additional fi nite $\left(q_{\mathrm{L}} \bar{\mu}\right.$ independent) contribution in the flavor singlet case. $Z_{S, \text { singlet }}$ is 
seen to be equal to the fermion mass renormalization $Z_{m}$, which is essential in the computation of quark masses. We note also that the scalar and pseudoscalar factors, necessary to convert flavor singlets from the $R I^{\prime}$ to the $\overline{M S}$ scheme, stay the same as their non-singlet counterparts.

The 2-loop computation of the renormalization functions for the Vector, Axial and Tensor bilinears is work currently in progress.

Besides the strictly local defi nitions of fermion bilinears, $\bar{\psi} \Gamma \psi$, one can consider a family of more extended operators, with the same classical continuum limit, as dictated by $\mathscr{O}\left(a_{\mathrm{L}}\right) \mathrm{im}-$ provement. The renormalization of these extended operators involves more Feynman diagrams; however, the computation is actually less cumbersome, since all additional contributions are now free of superfi cial divergences. We will report the results of this computation in a future work.

Acknowledgements: We would like to thank J. A. Gracey and S. A. Larin for private communication regarding their continuum results.

\section{References}

[1] A. Skouroupathis and H. Panagopoulos, Higher loop renormalization of fermion bilinear operators, submitted to Phys. Rev. D [arXiv:0707.2906].

[2] J.A. Gracey, Three loop anomalous dimension of non-singlet quark currents in the RI' scheme, Nucl. Phys. B662 (2003) 247 and private communication [hep-ph / 0304113 ] .

[3] F. Di Renzo, V. Miccio, L. Scorzato and C. Torrero, High-loop perturbative renormalization constants for Lattice QCD (I): finite constants for Wilson quark currents, [hep-lat/0611013] .

[4] Q. Mason et al., High-precision determination of the light-quark masses from realistic lattice QCD, Phys. Rev. D73 (2006) 114501 [hep-ph/0511160].

[5] S. Capitani et al., Renormalisation and off-shell improvement in lattice perturbation theory, Nucl. Phys. B593 (2001) 183 [hep-lat/ 0007004 ].

[6] A. Bode and H. Panagopoulos, The three-loop beta-function of QCD with the clover action, Nucl. Phys. B625 (2002) 198 [hep-lat/0110211].

[7] G. Martinelli et al., A General Method for Non-Perturbative Renormalization of Lattice Operators, Nucl. Phys. B445 (1995) 81 [hep-lat/9411010].

[8] E. Franco and V. Lubicz, Quark Mass Renormalization in the $\overline{M S}$ and RI schemes up to the NNLO order, Nucl. Phys. B531 (1998) 641 [hep-lat/9803491] .

[9] K.G. Chetyrkin and A. Rétey, Renormalization and Running of Quark Mass and Field in the Regularization Invariant and MS-bar Schemes at Three and Four Loops, Nucl. Phys. $\mathbf{B 5 8 3}$ (2000) 3 [hep-ph/9910332].

[10] H. Panagopoulos, A. Skouroupathis and A. Tsapalis, Free Energy and Plaquette expectation value for gluons on the lattice, in three dimensions, Phys. Rev. D73 (2006) 054511 [hep-lat/ 0601009 ] .

[11] S.A. Larin, The renormalization of the axial anomaly in dimensional regularization, Phys. Lett. $\mathbf{B 3 0 3}$ (1993) 113 and private communication [hep-ph/9302240, containing an extra section]. 